\title{
REDUÇÃO DO ESCURECIMENTO DO PERICARPO DE LICHIA COM ÁCIDO CLORÍDRICO ${ }^{1}$
}

\author{
LUIZ CARLOS CHAMHUM SALOMÃO², DANIELLE FABÍOLA PEREIRA SILVA ${ }^{3}$, \\ ALINE ROCHA ${ }^{4}$, ROBSON RIBEIRO ALVES ${ }^{5}$, ROSANA GONÇALVES PIRES MATIAS ${ }^{5}$
}

RESUMO - Foram avaliados os efeitos da imersão em soluções com diferentes concentrações de ácido clorídrico $(\mathrm{HCl})$ sobre a redução do escurecimento do pericarpo e a conservação pós-colheita de lichia. Lichias 'Bengal' foram colhidas com o pericarpo completamente vermelho e imersas por dois minutos em 0 (controle); 0,25; 0,5; 1,0 e 2,0 M de $\mathrm{HCl}$. Após os tratamentos, os frutos foram acondicionados em bandejas de poliestireno expandido e recobertos com filme de policloreto de vinila (PVC). Os frutos foram armazenados em câmara fria a $10,0 \pm 0,9^{\circ} \mathrm{C}$ e $90 \pm 5 \%$ de UR e avaliados de 4 em 4 dias. Após 24 dias de armazenamento, os frutos de todos os tratamentos apresentaram perda de massa fresca em torno de 4,5\%. Até o $16^{\circ}$ dia de armazenamento, os frutos tratados com $\mathrm{HCl}$ 2,0 M não apresentaram alteração significativa na coloração do pericarpo $\left(\Delta \mathrm{E}, \mathrm{h}\right.$ e $\left.\mathrm{L}^{*}\right)$ em relação ao dia da colheita. Visualmente, esses frutos permaneceram sem alterações perceptíveis na coloração, até os 24 dias de armazenamento refrigerado. Naqueles não tratados (controle) e nos tratados com $\mathrm{HCl}$ a $0,25 \mathrm{M}$ e $0,5 \mathrm{M}$, o escurecimento do pericarpo iniciou-se no $4^{\circ}$ dia de armazenamento refrigerado.

Termos para indexação: Litchi chinensis Sonn., cor da casca, armazenamento refrigerado.

\section{REDUCTION OF THE PERICARP BROWNING OF LYCHEE WITH HYDROCHLORIC ACID}

\begin{abstract}
This study assessed the effects of immersion in different concentrations of hydrochloric acid $(\mathrm{HCl})$ on pericarp color retention and postharvest conservation of lychees. 'Bengal' lychees were harvested with completely red pericarp and immersed for two minutes in $\mathrm{HCl}$ at concentrations of 0 (control), 0.25 , $0.5,1$ and $2 \mathrm{M}$. After the treatments, fruits were packed in expanded polystyrene trays and covered with polyvinyl chloride film (PVC). The fruits were stored in cold chamber at $10 \pm 0.9^{\circ} \mathrm{C}$ and $90 \pm 5 \% \mathrm{RH}$. The fruits were evaluated every 4 days over a period of 24 days. After 24 days of storage, fruits of all treatments showed weight loss of around $4.5 \%$. By the $16^{\text {th }}$ day of storage, fruits treated with $2 \mathrm{M} \mathrm{HCl}$ showed nonsignificant changes in pericarp color $(\Delta \mathrm{E}$, Hue angle and luminosity) in relation to the harvest day. These fruits remained without visually perceptible change in color up to 24 days of cold storage. Pericarp darkening began on the $4^{\text {th }}$ day of cold storage in fruits untreated (control) and treated with 0.25 and $0.5 \mathrm{M}$ of $\mathrm{HCl}$.
\end{abstract}

Index terms: Litchi chinensis Sonn., skin color, cold storage.

\footnotetext{
1(Trabalho 156-11). Recebido em: 16-05-2011. Aceito para publicação em: 06-03-2012.

${ }^{2}$ Eng Agr. DSc. - Prof. Departamento de Fitotecnia - Universidade Federal de Viçosa - CEP 36.570-000 - Viçosa-MG- Brasil 1salomao@ufv.br

${ }^{3}$ Eng Agr ${ }^{a}$. DSc - Pós-Doutoranda (PNPD-CAPES) - Departamento de Fitotecnia - Universidade Federal de Viçosa - CEP 36.570-000 - Viçosa-MG - Brasil - danieele@ufv.br

${ }^{4}$ Eng Agr ${ }^{\mathrm{a}}$. DSc. - Prof ${ }^{\mathrm{a}}$. Instituto Federal de Educação, Ciência e Tecnologia do Sertão Pernambucano (IFET Petrolina), Petrolina-PE - Brasil - rochaline@hotmail.com

${ }^{5}$ Estudantes do Programa de Pós-Graduação em Fitotecnia - Departamento de Fitotecnia - Universidade Federal de Viçosa - CEP 36.570-000 - Viçosa-MG - Brasil, robson@ufv.br, rosana.pires@ufv.br
} 


\section{INTRODUÇÃO}

A lichia (Litchi chinensis Sonn.) é um fruto de elevado valor comercial, por essa razão a viabilização de seu cultivo pode gerar um importante acréscimo de renda para os produtores (PICOLI et al., 2010). No entanto, perde rapidamente sua atrativa coloração vermelha após a colheita devido ao rápido escurecimento do pericarpo, causado tanto pela perda de água quanto pela atividade de enzimas oxidativas (SILVA et al., 2010), assim como pela degradação da antocianina (HOJO et al., 2011).

A oxidação do ácido ascórbico aumenta a degradação da antocianina. Os mecanismos desta reação não estão bem esclarecidos, mas Underhill (1992) sugere que o peróxido de hidrogênio produzido pela degradação do ácido ascórbico participa da oxidação da antocianina.

Duas estratégias principais têm sido empregadas para reduzir o escurecimento do pericarpo: a redução da perda de umidade e a supressão do escurecimento por tratamentos físicos e químicos (SOUZA et al., 2010).

O escurecimento enzimático do pericarpo da lichia é o primeiro sinal visível do declínio da qualidade do fruto. A atividade das polifenoloxidases (PPO) está estritamente ligada às mudanças de coloração devido à formação de polímeros coloridos (SOUZA et al., 2010). Dessa forma, medidas de coloração podem ser consideradas como índice indireto da atividade das PPOs. Amostras que não apresentam acastanhamento ou outras cores anômalas são consideradas livres da atividade da PPO (SAENGNIL et al., 2006).

Vários tratamentos têm sido sugeridos visando à redução do escurecimento em lichia. $\mathrm{O}$ uso de ácidos como o ascórbico e o cítrico tem sido recomendado devido ao seu efeito na estabilidade da cor de antocianinas e na atividade de oxidases (HOJO et al., 2011).

A imersão dos frutos em soluções diluídas de $\mathrm{HCl}$ tem possibilitado o restabelecimento da coloração pela conversão de pigmentos escuros a íons flavino, que são predominantes em $\mathrm{pH}$ baixo. A imersão dos frutos em $\mathrm{HCl}$ a $1 \mathrm{~N}$ por dois minutos resultou em completa recuperação da coloração dentro de 24 a 48 horas após o tratamento (UNDERHILL, 1992).

De acordo com Lima et al. (2010), o índice de escurecimento da lichia aumenta durante o armazenamento à temperatura ambiente, assim como a concentração de antocianina diminui marcadamente; sendo assim, o escurecimento tem sido atribuído à degradação da antocianina devido à ação das enzimas oxidativas, polifenoloxidase (PPO), peroxidase
(POD) e ácido ascórbico oxidase.

A imersão em lecitina e ceras, tratamentos com compostos à base de enxofre, e acondicionamento em embalagens plásticas (SILVA et al., 2010; TAYLOR, 1993) também têm sido utilizados para a conservação da coloração. Já, a redução da perda de umidade é obtida com o revestimento dos frutos com filmes plásticos, a exemplo de cloreto de polivinila ou PVC (UNDERHILL, 1992).

Vários trabalhos foram realizados utilizando diferentes técnicas tais como imersão em ácido ascórbico associado à atmosfera modificada (SILVA et al., 2010); pré-resfriamento (SAAVEDRA DEL AGUILA et al., 2009 a); imersão em 4- hexilresorcinol, ácido cítrico e ácido ascórbico (SAAVEDRA DEL AGUILA ET al., 2009 b); embalagens associadas a refrigeração (CHEN et al., 2001); imersão em ácido oxálico (SAENGNIL et al., 2006) e tratamento térmico (SOUZA et al., 2010), todos com o objetivo de reduzir o escurecimento do pericarpo e de manter a qualidade pós-colheita de lichia. Todavia, de acordo com esses trabalhos, as lichias deterioram-se rapidamente, não sendo possível armazenar os frutos por períodos superiores a sete dias, mesmo sob armazenamento refrigerado.

Este trabalho teve como objetivo avaliar os efeitos de diferentes concentrações de ácido clorídrico $(\mathrm{HCl})$ sobre a redução do escurecimento do pericarpo e a conservação pós-colheita de lichia 'Bengal'.

\section{MATERIAL E MÉTODOS}

Frutos de lichieira 'Bengal' com pericarpo completamente vermelho $\left(L^{*}=49,57, a^{*}=27,64\right.$, $\left.\mathrm{b}^{*}=11,21, \mathrm{C}=29,96 \mathrm{e} \mathrm{h}=22,1\right)$ foram colhidos, no período da manhã, em dezembro de 2008, de plantas do Pomar Experimental da Universidade Federal de Viçosa, em Viçosa, Minas Gerais (21 $07^{\circ} \mathrm{S}, 4^{\circ} 57^{\prime} \mathrm{W}$, $651 \mathrm{~m}$ de altitude).

Após a colheita, foram selecionados os frutos com pericarpo uniformemente vermelho. Na sequência, os frutos foram imersos em solução-controle e com diferentes concentrações de ácido clorídrico $(0,25 ; 0,5 ; 1,0$ e $2,0 \mathrm{M})$ por 2 minutos, secos à temperatura ambiente, acondicionados em bandejas de poliestireno expandido (220 $\mathrm{mm}$ x $140 \mathrm{~mm}$ x $40 \mathrm{~mm})$ e recobertos com filme de policloreto de vinila (PVC) de $14 \mu \mathrm{m}$ de espessura, armazenados em câmara fria a $10,0 \pm 0,9^{\circ} \mathrm{C}$ e $90 \pm 5 \%$ de UR e avaliados a cada 4 dias, durante 24 dias. A temperatura de $10^{\circ} \mathrm{C}$ foi utilizada para simular o comércio. O ácido clorídrico utilizado apresentava normalidade de $11,74 \mathrm{Eq} \mathrm{L}^{-1}$,e os pHs das concentrações utilizadas foram: 6,98 ; 
0,$87 ; 0,50 ; 0,34$ e 0,14 para os tratamentos-controle, 0,$25 ; 0,50 ; 1,0$ e $2,0 \mathrm{M}$, respectivamente.

Foram avaliados quanto à perda de massa fresca, atributos de coloração do pericarpo, teor de sólidos solúveis e acidez titulável da polpa, assim como de ácido ascórbico do pericarpo e da polpa. A perda de massa fresca foi determinada por gravimetria, sendo os resultados expressos em porcentagem de perda de massa.

A coloração do pericarpo foi determinada usando-se colorimetro Minolta (Color Reader CR10). Foram feitas duas leituras por fruto em posições diametralmente opostas. A partir das leituras de $\mathrm{L}^{*}$ (luminosidade), $\mathrm{a}^{*}$ e $\mathrm{b}^{*}$, foram determinados a diferença de coloração $(\Delta \mathrm{E})$ e o ângulo hue $(\mathrm{h})$ (MCGUIRRE, 1992).

O teor de sólidos solúveis da polpa foi determinado com o auxílio de um refratômetro digital em amostras de polpa trituradas em liquidificador (Official methods of analysis of the Association of Official Analytical Chemists International, 1997). A acidez titulável foi determinada por titulação com $\mathrm{NaOH}$ a $0,1 \mathrm{M}$ e expressa em porcentagem de ácido málico (AOAC, 1997). O teor de ácido ascórbico do pericarpo e da polpa foi determinado por titulação com reagente de Tillman [2,6 diclorofenolindofenol (sal sódico) a 0,1\%] (AOAC, 1997). Os resultados foram expressos em $\mathrm{mg} 100 \mathrm{~g}^{-1}$ de polpa.

Utilizou-se o delineamento estatístico inteiramente casualizado, disposto em esquema fatorial $5 \times 7$, com três repetições, em que o primeiro fator correspondeu às doses de ácido clorídrico $(0 ; 0,25$; 0,$5 ; 1,0$ e 2,0 M), e o segundo fator, aos períodos de amostragem $(0 ; 4 ; 8 ; 12 ; 16 ; 20$ e 24 dias $)$. Cada parcela foi composta por cinco frutos, mantidos em bandejas de poliestireno expandido e recobertos com filme PVC. Os dados foram analisados por meio das análises de variância e regressão, usando-se o programa SAEG 9.1 - Sistema para Análises Estatísticas e Genéticas (SAEG, 2007).

\section{RESULTADOS E DISCUSSÃO}

A perda de massa fresca diária média foi de $0,169 \%$ e não variou com o aumento da concentração de ácido clorídrico (Figura 1A). A perda de massa média acumulada nos 24 dias foi de 4,06\% para o controle. Para Saavedra Del Aguila et al. (2009 a), perdas de massa na ordem de 3 a $5 \%$ causam escurecimento do pericarpo. Por outro lado, Chen et al. (2001) consideraram que o escurecimento do pericarpo de lichia só ocorre com perda de massa fresca superior a 7,6\%, enquanto o escurecimento total do pericarpo é observado com perdas superiores a 18,2\%.
Os tratamentos com $1 \mathrm{M}$ e $2 \mathrm{M}$ foram eficientes em reduzir o escurecimento do pericarpo da lichia durante o período experimental. Na Figura $1 \mathrm{~B}$, tem-se que, no $24^{\circ}$ dia de armazenamento para os frutos do tratamento-controle, a diferença de coloração em relação ao dia da colheita foi de 16,65, enquanto nos tratados com $1 \mathrm{M}$ e $2 \mathrm{M}$, a diferença de coloração foi de apenas 8,59 e 6,81, respectivamente, o que demonstra o potencial do $\mathrm{HCl}$ em prevenir o escurecimento do pericarpo de lichia por período prolongado (24 dias).

Silva et al. (2010) também observaram que, em frutos de lichieira 'Bengal' imersos em soluções de ácido ascórbico a $0 ; 5 ; 10 ; 15$ e $30 \mathrm{mM}$, ocorreu escurecimento do pericarpo, independentemente da dose utilizada, provavelmente pelo ácido ascórbico ser um ácido fraco quando comparado ao $\mathrm{HCl}$, e o ácido ascórbico pode atuar como agente pró-oxidante, ou seja, os produtos gerados pela degradação do ácido ascórbico são também responsáveis pela formação de pigmentos escuros da casca (UNDERHILL, 1992).

O ângulo hue apresentou valores de $39,77^{\circ}$; $41,51^{\circ} ; 46,32^{\circ} ; 32,56^{\circ}$ e $26,12^{\circ}$ para as concentrações de $0 ; 0,25 ; 0,5 ; 1,0$ e 2,0 M, respectivamente, no $16^{\circ}$ dia de armazenamento (Figura $1 \mathrm{C}$ ), indicando que os frutos tratados com $1 \mathrm{M}$ e $2 \mathrm{M}$ de $\mathrm{HCl}$ mantiveram a coloração vermelha mais próxima daquela do dia da colheita $\left(\mathrm{h}=24,30^{\circ}\right)$.

Observou-se, durante todo o período experimental, redução intensa nos valores de $L^{*}$ dos frutos-controle (0 M), que passaram de 49,01, no dia zero, para 41,02, no $24^{\circ}$ dia de armazenamento, indicando haver escurecimento do pericarpo. Os valores médios de L* reduziram-se de 49,38; 49,86; 50,0 e 50,3 no dia zero, para 44,04; 44,71; 46,89 e 47,29 no $24^{\circ}$ dia de armazenamento para as doses 0,$25 ; 0,5 ; 1,0$ e 2,0M, respectivamente (Figura 1D). Saavedra del Aguila et al. (2009 b) relataram que nenhum tratamento por imersão em solução aquosa contendo antioxidante $\left(300 \mathrm{mg} . \mathrm{L}^{-1}\right.$ de 4 - hexilresorcinol, ou $300 \mathrm{mg} \mathrm{L}^{-1}$ de ácido ascórbico, ou $300 \mathrm{mg}$ $\mathrm{L}^{-1}$ de ácido cítrico, ou $300 \mathrm{mg} \mathrm{L}^{-1}$ de ácido cítrico + $300 \mathrm{mg} \mathrm{L}^{-1}$ de ácido ascórbico) conseguiu reduzir o escurecimento do pericarpo de lichias armazenadas a $5^{\circ} \mathrm{C}$. Estes autores afirmaram que a mudança indesejável na coloração da lichia, indicada por rápido decréscimo da luminosidade ( $\left.\mathrm{L}^{*}\right)$, indica escurecimento e perda da coloração vermelho-intensa do pericarpo, cuja consequência imediata é a perda de seu valor comercial.

A variação na coloração vermelha, para uma tonalidade marrom, em frutos colhidos, pode ocorrer em poucas horas, ou no máximo em três 
dias após a colheita, em temperatura de 20 a $25^{\circ} \mathrm{C}$ (SOUZA et al., 2010). Neste trabalho, as características de coloração $\left(\Delta \mathrm{E}, \mathrm{h}\right.$ e $\left.\mathrm{L}^{*}\right)$ indicaram que o $\mathrm{HCl}$ nas concentrações de $1 \mathrm{M}$ e $2 \mathrm{M}$ reduziu de forma significativa a perda da coloração vermelha do pericarpo da lichia até o $12^{\circ}$ e $24^{\circ}$ dia de armazenamento, respectivamente (Figuras 1B, 1C e 1D). Com base nas características de coloração avaliadas $(\Delta \mathrm{E}, \mathrm{h}$ e $\mathrm{L})$, não foi possível observar qualquer alteração na coloração do pericarpo dos frutos imersos em $\mathrm{HCl} 2 \mathrm{M}$ durante todo o período experimental. Nas concentrações de 0,25 e $0,5 \mathrm{M}$, o $\mathrm{HCl}$ acentuou o escurecimento do pericarpo, em quatro dias de armazenamento.

Observou-se redução nos teores de sólidos solúveis nos tratamentos com as diferentes concentrações de $\mathrm{HCl}$ estudadas (Figura 2A). Resultados semelhantes foram encontrados por Silva et al. (2010) que, trabalhando com diferenças concentrações de ácido ascórbico em lichia, observaram que houve queda nos teores de sólidos solúveis para todas as concentrações estudadas.

A acidez titulável também decresceu durante o período experimental em todos os tratamentos (Figura 2B). Resultados semelhantes foram encontrados por Pesis et al. (2002), em lichias 'Mauritius' acondicionadas em sacolas de polietileno e armazenadas sob refrigeração; por Mahajan e Goswami (2004), em lichias 'Bombay' armazenadas a $2^{\circ} \mathrm{C}$, sob atmosfera controlada; por Wu et al. (2001), em lichias 'Feizixiao' embaladas em polietileno de 30 $\mu \mathrm{m}$ e armazenadas a $4^{\circ} \mathrm{C}$ por 27 dias; e por Chaiprasart (2005), em lichias 'Hong Huai'embaladas em filmes de polietileno e $\mathrm{PVC}$, a $5^{\circ} \mathrm{C}$, por 12 dias.

$\mathrm{O}$ teor de ácido ascórbico do pericarpo da lichia apresentou ajuste quadrático para todas as concentrações de ácido clorídrico, com tendência de redução durante o período de armazenamento
(Figura 3A). Para a dose $0 \mathrm{M}$ (controle), observou-se o maior decréscimo, chegando ao 24 o dia de armazenamento com perda de $75,56 \%$ de ácido ascórbico. Todavia, nos frutos tratados com $1 \mathrm{M}$ e $2 \mathrm{M}$ de $\mathrm{HCl}$, as perdas registradas de ácido ascórbico, no $24^{\circ}$ dia, foram de $40,37 \%$ e $31,90 \%$, respectivamente.

De acordo com Underhill (1992), o ácido ascórbico previne o escurecimento enzimático em alimentos, sendo, entretanto, consumido durante o processo. Sua eficiência na retenção da coloração do pericarpo da lichia não é alta, uma vez que seu efeito inibitório sobre as enzimas é reversível. Nota-se que o $\mathrm{HCl}$ a $1 \mathrm{M}$ e a $2 \mathrm{M}$ foi eficiente em manter os teores de ácido ascórbico no pericarpo das lichias próximos aos valores iniciais. Os frutos destes tratamentos apresentaram menor escurecimento do pericarpo (índices de coloração), mostrando ter relação direta com a prevenção do escurecimento. Isso porque o ácido ascórbico pode atuar como agente pró-oxidante, ou seja, os produtos gerados pela degradação do ácido ascórbico são também responsáveis pela formação de pigmentos escuros da casca (UNDERHILL, 1992).

O HCl também foi eficiente em manter elevado o teor de ácido ascórbico da polpa de lichia (Figura 3B). Observou-se, no 24 dia de armazenamento, que os frutos do tratamento controle tinham redução de $67,45 \%$ em seu teor, em relação ao dia zero. Por outro lado, para frutos tratados com $1 \mathrm{M} \mathrm{e} 2 \mathrm{M}$ de $\mathrm{HCl}$, este decréscimo foi de $41,23 \%$ e $34,92 \%$ do teor de ácido ascórbico, respectivamente. Isto indica que o $\mathrm{HCl}$ é eficiente não só em conservar a coloração do pericarpo, mas também em minimizar a perda de qualidade pós-colheita do fruto. 

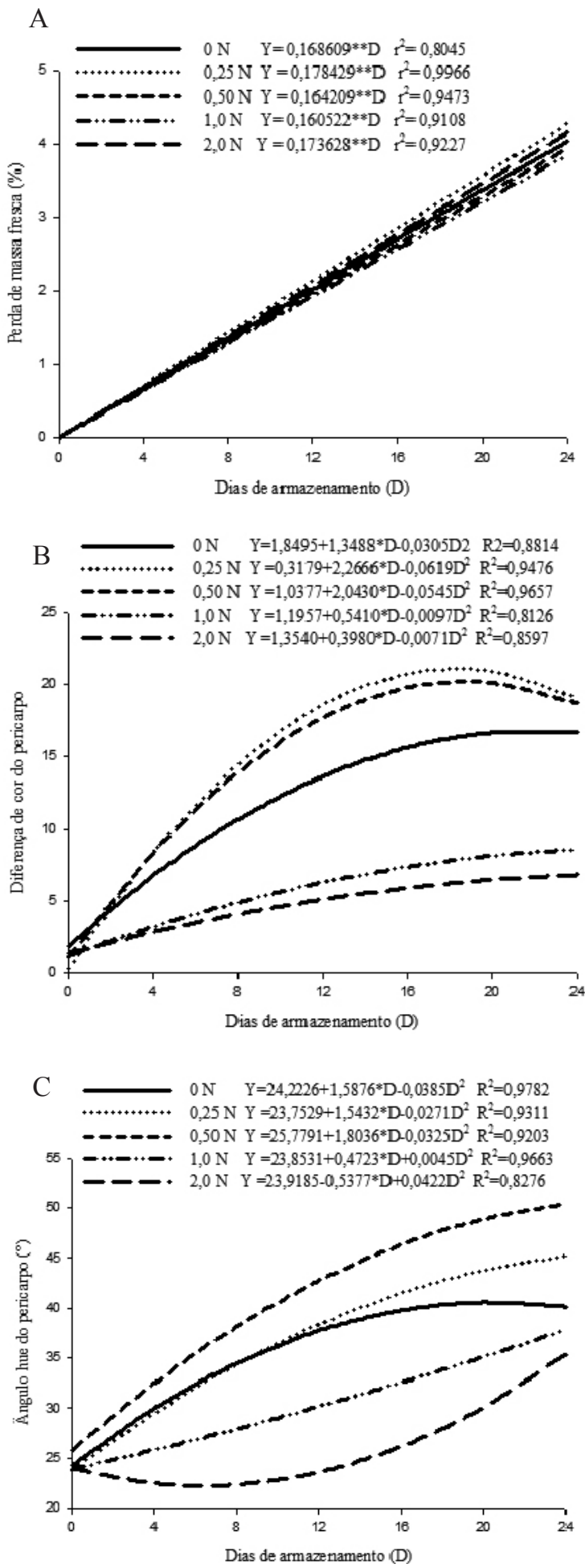

continua... 


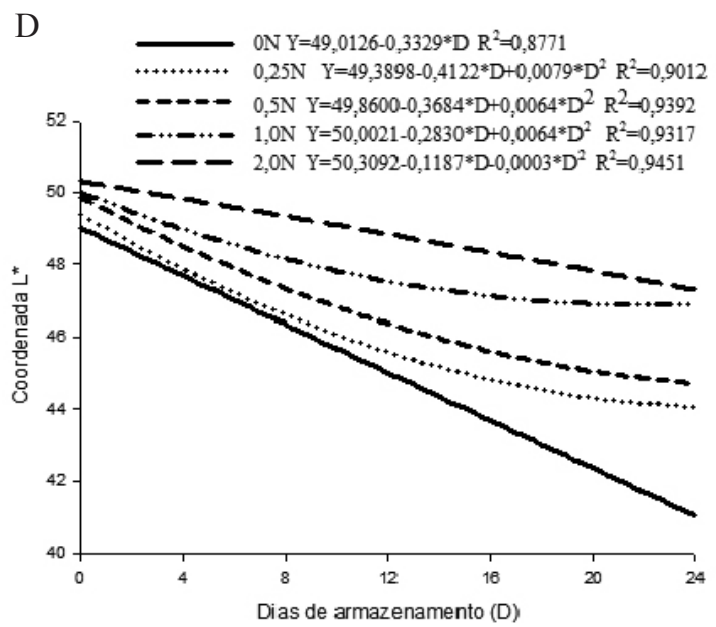

FIGURA 1- Perda de massa fresca do fruto (A), diferença de coloração ou $\Delta \mathrm{E}$ (B), ângulo hue (C) e luminosidade $\left(\mathrm{L}^{*}\right)$ do pericarpo (D), em lichias 'Bengal' tratadas com diferentes concentrações de ácido clorídrico e armazenadas a $10^{\circ} \mathrm{C}$ e $90 \%$ de UR.
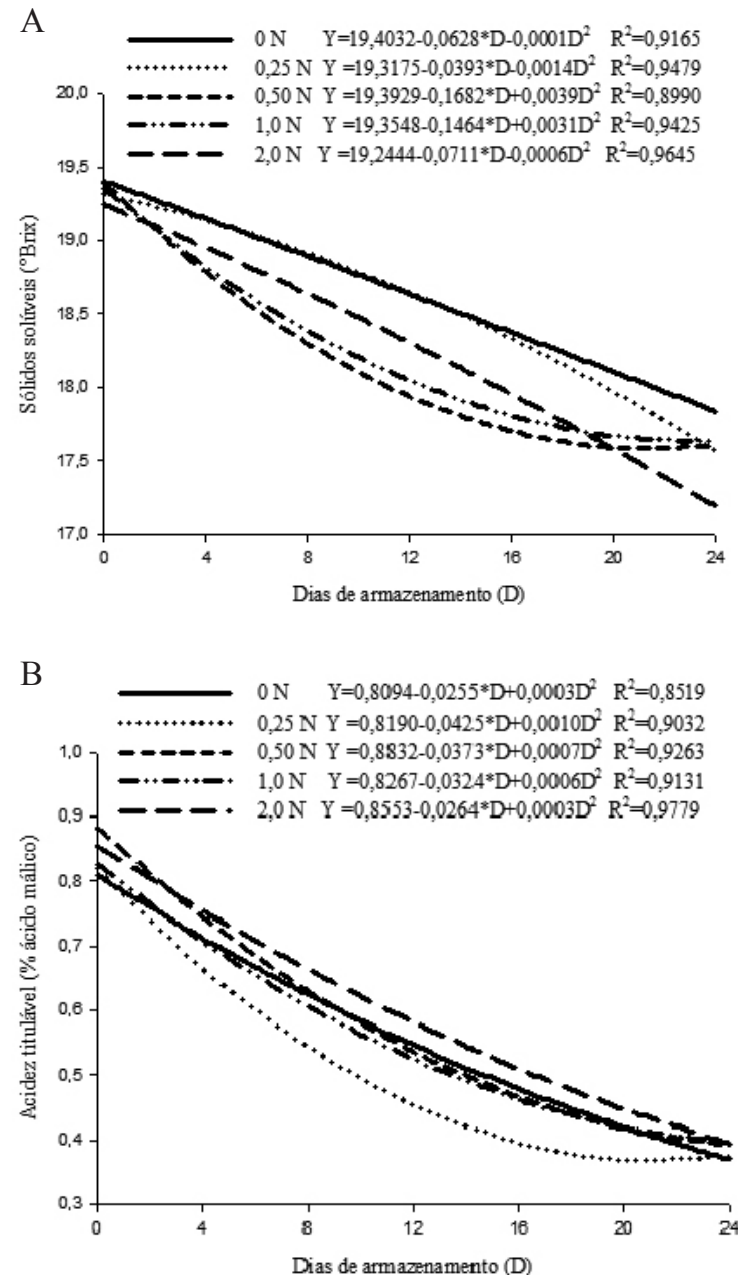

FIGURA 2- Teor de sólidos solúveis (A) e acidez titulável (B) em lichias 'Bengal' tratadas com diferentes concentrações de ácido clorídrico e armazenadas a $10^{\circ} \mathrm{C}$ e $90 \%$ de UR. 

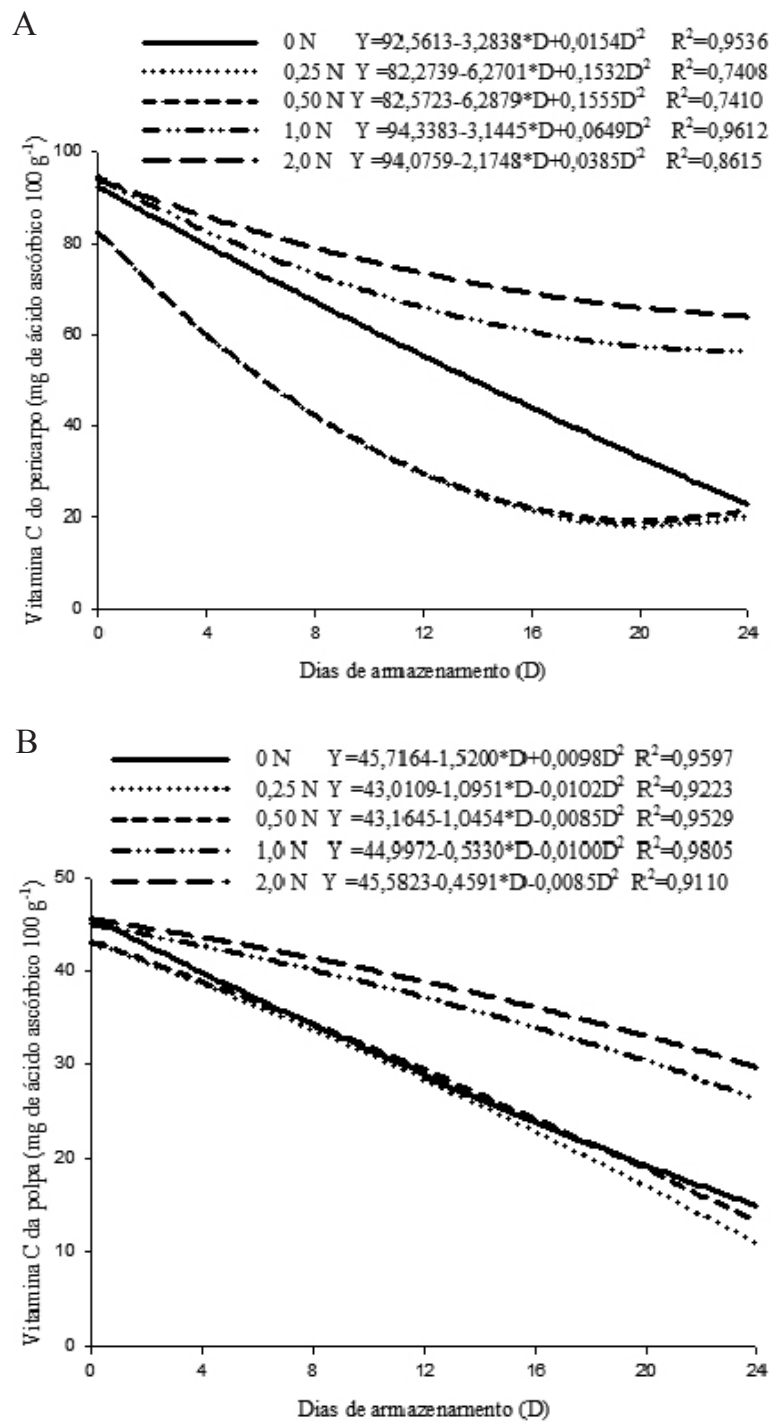

FIGURA 3 - Teores de ácido ascórbico no pericarpo (A) e na polpa (B) de lichias 'Bengal' tratadas com diferentes concentrações de ácido clorídrico e armazenadas a $10^{\circ} \mathrm{C}$ e $90 \%$ de UR.

\section{CONCLUSÕES}

Os tratamentos por imersão em soluções contendo $1 \mathrm{M}$ ou $2 \mathrm{M}$ de $\mathrm{HCl}$ são eficientes em reduzir o escurecimento do pericarpo de Lichia, sem afetar as características físicas e químicas avaliadas.

\section{AGRADECIMENTOS}

Ao Conselho Nacional de Desenvolvimento Científico e Tecnológico - CNPq, à Coordenação de Aperfeiçoamento de Pessoal de Nível Superior-CAPES, e à Fundação de Amparo à Pesquisa do Estado de Minas Gerais - FAPEMIG, pelo apoio financeiro.

\section{REFERÊNCIAS}

AOAC. Official methods of analysis of the Association of Official Analytical Chemists International. $16^{\text {th }}$ ed. Washington, 1997. v.2, p.37-10, 42-2, 44-3, 45-16.

CHAIPRASART. P. Effect of modified atmosphere packaging by $\mathrm{PE}$ and $\mathrm{PVC}$ on quality changes of lychee fruits. Acta Horticulturae, The Hague, n. 665, p. 373-380, 2005.

CHEN, W.; WU, Z.; SU, M. Postharvest research and handling of litchi in China - a review. Acta Horticulturae, The Hague, n. 558, p. 321- 329, 2001. 
HOJO, E. T. D.; DURIGAN, J. F.; HOJO, R. H.; DONADON, J. R.; MARTINS, R. N. Uso de tratamento hidrotérmico e ácido clorídrico na qualidade de lichia 'Bengal'. Revista Brasileira de Fruticultura, Jaboticabal, v.33, n. 2, p. 386-393, 2011.

LIMA, R. A. Z.; C ABREU, C.M. P. de; ASMAR, S. A.; CORRÊA, A. D.; SANTOS, C. D. dos. Embalagens e recobrimento em lichias (Litchi chinensis sonn.) armazenadas sob condições não controladas. Ciência e Agrotecnologia, Lavras, v.34, n.4, p.914921, 2010.

MAHAJAN, P. V.; GOSWAMI, T. K. Extended storage life of litchi fruit using controlled atmosphere and low temperature. Journal of Food Processing and Preservation, Westport, v. 28, p. 388-403, 2004.

McGUIRE, R. G. Reporting of objective color measurements. HortScience, Alexandria, v. 27, n. 12, p. 1254-1260, 1992.

MINOLTA CORP. Precise color communication: color control from feeling to instrumentation. Ramsey: Minolta Corporation Instrument Systems Division, 1994. 49 p.

PICOLI, P. R. F.; VIEIRA, M. R.; SILVA, E. A. da; MOTA, M. S. de O. da. Ácaros predadores associados ao ácaro-da-erinose da lichia. Pesquisa Agropecuária Brasileira, Brasília, v.45, n.11, p. 1246-1252, 2010.

PESIS, E.; DVIR, O.; FEYGENBERG, O.; ARIE, R. B.; ACKERMAN, M.; LICHTER, A. Production of acetaldehyde and ethanol during maturation and modified atmosphere storage of litchi fruit. Postharvest Biology and Technology, Amsterdam, v. 26, p. 157-165, 2002.

SAEG. Sistema para análises estatísticas, versão 9.1. Viçosa: Fundação Arthur Bernardes, UFV, 2007. CD-ROM.
SAENGNIL，K.; LUEANGPRASERT，K.; UTHAIBUTRA, J. Control of enzymatic browning of harvested 'Hong Huay' litchi fruit with hot water and oxalic acid dips Science Asia, Shangai, v.32, p.345-350, 2006.

SAAVEDRA DEL AGUILA, J.; HOFMAN, P.; CAMPBELL, T.; MARQUES, J. R.; HEIFFIG-DEL AGUILA, L. S.; KLUGE, R. A. Pré-resfriamento em água de lichia 'B3' mantida em armazenamento refrigerado. Ciência Rural, Santa Maria, v. 39, n. 8, p. 2373-2379, 2009a.

SAAVEDRA DEL AGUILA, J.; HEIFFIG-DEL AGUILA, L.S.; SASAKI, F.F.; ORTEGA, E.M.M.; KLUGE, R.A. Efeito de antioxidantes na taxa respiratória e na produção de etileno de lichia 'Bengal' armazenada sob refrigeração. Revista Iberoamericana de Tecnologia Postcosecha, México, v. 10, p. 8-13, 2009b.

SILVA, D. F. P.; CABRINI, E. C.; ALVES, R. R.; SALOMÃO, L.C.C. Uso do ácido ascórbico no controle do escurecimento do pericarpo de lichia. Revista Brasileira de Fruticultura, Jaboticabal, v. 32, n. 2, p. 618-627, 2010.

SOUZA, A. V.; VIEITES, R. L.; KOHATSU, D. S.; LIMA, G. P. P. Tratamento térmico na manutenção da coloração de lichias. Revista Brasileira de Fruticultura, Jaboticabal, v. 32, n. 1, p. 067-073, 2010.

TAYLOR, J. E. Exotics. In: SEYMOUR, G. B.; TAYLOR, J. E.; TUCKER, G. A. Biochemistry of fruit ripening. Cambridge: Chapman e Hall, 1993. p.151-187.

UNDERHILL, S. J. R. Lychee (Litchi chinensis Sonn.) pericarp browning. Tropical Science, London, v.32, n.3, p.305-312, 1992.

WU, Z.; SU, M.J.I.; CHEN, W.; HAN, D. A study of the behavior of 'Feizixiao' litchi during storage. Acta Horticulturae, The Hague, v. 558, p. 381-386, 2001. 